\title{
RETRACTED ARTICLE: Upregulation of miR-21 and downregulation of miR-494 may serve as emerging molecular biomarkers for prediagnostic samples of subjects who developed nasopharyngeal carcinoma associates with lymph node metastasis and poor prognosis
}

\author{
Masoomeh Dadpay ${ }^{1}$ - Mojtaba Zarea ${ }^{2}$. Rahman Ghaffarzadegan Rabati ${ }^{3}$. \\ Bijan Rezakhaniha $^{4} \cdot$ Babak Barari $^{5} \cdot$ Vahid Behnod $^{6} \cdot$ Katayoun Ziari $^{7}$
}

Published online: 21 August 2015

(C) International Society of Oncology and BioMarkers (ISOBM) 2016

\section{RETRACTED ARTICLE: Tumor Biol.}

DOI 10.1007/s13277-015-3905-1

This article has been retracted at the request of the Editor-in-Chief, the International Society of Oncology and BioMarkers (ISOBM) and the Publisher per the Committee on Publication Ethics guidelines. The article shows evidence of irregularities in authorship during the submission process, there is strong reason to believe that the peer review process was compromised and there are similarities

Electronic supplementary material The online version of this article (doi:10.1007/s13277-015-3905-1.) contains supplementary material, which is available to authorized users.

Katayoun Ziari

drk_ziari@yahoo.com

1 Department of Pathology, Imam Reza Hospital, AJA University of Medical Sciences, Tehran, Iran

2 Center for Chemical Biology, Indian Institute of Chemical Technology (IICT), Tarnaka, Hyderabad, India

3 Shahid Abbas Abdollahi, Molecular Biology Research Center, Shahid Mahallati Hospital, Tabriz, Iran

4 Department of Urology, Imam Reza Hospital, AJA University of Medical Sciences, Tehran, Iran

5 Dr Genetic Medical, Tehran University of Medical Sciences, Tehran, Iran

6 Baqiyatallah University of Medical Sciences, Tehran, Iran

7 Department of Pathology, Be'sat Hospital, AJA University of Medical Sciences, Tehran, Iran with the following articles which were all submitted within a close timeframe:

Yasan Sadeghian, Zahra Kamyabi-Moghaddam, Seyed Mohamad Hossein Tabatabaei Nodushan, Samaneh Khoshbakht, Behnam Pedram, Emad Yahaghi, Aram Mokarizadeh, Mahdi Mohebbi, Profiles of tissue microRNAs; miR-148b and miR-25 serve as potential prognostic biomarkers for hepatocellular carcinoma. Tumor Biol. First Online: 25 July 2015 DOI: 10.1007/s13277-015-3799-y

Date received: 1 July 2015

Afshin Taheri Azam, Reza Bahador, Hamid Hesarikia, Mohammadreza Shakeri, Ali Yeganeh, Down-regulation of microRNA-217 and microRNA-646 acts as potential predictor biomarkers in progression, metastasis and unfavorable prognosis of human osteosarcoma. Tumor Biol. First Online: 31 July 2015 10.1007/s13277-015-3821-4

Date received: 5 July 2015

The retracted article was received: 27 June 2015

As such the validity of the content of this article cannot be verified.

The online version of this article contains the full text of the retracted article as electronic supplementary material. 